\title{
ON THE MAJORANA CONDITION FOR NONLINEAR DIRAC SYSTEMS
}

\author{
TIMOTHY CANDY AND SEBASTIAN HERR
}

\begin{abstract}
For arbitrarily large initial data in an open set defined by an approximate Majorana condition, global existence and scattering results for solutions to the Dirac equation with Soler-type nonlinearity and the Dirac-KleinGordon system in critical spaces in spatial dimension three are established.
\end{abstract}

\section{INTRODUCTION}

Let $m, M \geq 0$. Using the summation convention with respect to $\mu=0, \ldots, 3$, the cubic Dirac equation (Soler model) for a spinor $\psi: \mathbb{R}^{1+3} \rightarrow \mathbb{C}^{4}$ is given by

$$
-i \gamma^{\mu} \partial_{\mu} \psi+M \psi=(\bar{\psi} \psi) \psi
$$

Here, $x^{0}=t, \partial_{0}=\partial_{t}$, and $\bar{\psi}=\psi^{\dagger} \gamma^{0}$ is the Dirac adjoint, where $\psi^{\dagger}$ denotes the complex conjugate transpose of the spinor $\psi$, and the matrices $\gamma^{\mu} \in \mathbb{C}^{4 \times 4}$ are the standard Dirac matrices, see [9]. Writing $\square=\partial_{t}^{2}-\Delta$, the Dirac-Klein-Gordon system is

$$
\begin{aligned}
-i \gamma^{\mu} \partial_{\mu} \psi+M \psi & =\phi \psi, \\
\square \phi+m^{2} \phi & =\bar{\psi} \psi,
\end{aligned}
$$

where $\phi: \mathbb{R}^{1+3} \rightarrow \mathbb{R}$ is a scalar field. These equations (1.1) and (1.2) arise as in relativistic quantum mechanics as toy models for interactions of elementary particles, see e.g. [6, 16.

In previous work, we have addressed the initial value problems for the above equations for small initial data of low regularity. Concerning the cubic Dirac equation, we have obtained small data global well-posedness and scattering in the massive case $M>0$ [3, 4] as well as the massless case $M=0$ [7. For the massive Dirac-Klein-Gordon system, we have obtained small data global well-posedness in the non-resonant regime for initial data of subcritical regularity [5] and both in the resonant and the non-resonant regime in the critical space with additional angular regularity 9 . Concerning a more complete account on earlier work on the low regularity well-posedness problem, we refer to the references therein. The purpose of the current article to gain insight into the asymptotic behaviour of an open set of large data solutions to (1.1) and (1.2).

2010 Mathematics Subject Classification. 42B37, 35Q41.

Key words and phrases. Cubic Dirac equation, Dirac-Klein-Gordon system, global existence, scattering, Majorana condition.

Financial support by the German Research Foundation through the CRC 1283 "Taming uncertainty and profiting from randomness and low regularity in analysis, stochastics and their applications" is acknowledged. 
In 10, Chadam and Glassey considered the equations (1.1) and (1.2) under the assumption that the initial data was of the form

$$
\psi(0)=\left(f, g,-g^{*}, f^{*}\right)^{t}
$$

where, given a complex scalar (or vector) $z \in \mathbb{C}^{n}$, we let $z^{*}$ denote the complex conjugate, and $f, g: \mathbb{R}^{3} \rightarrow \mathbb{C}$. This condition (1.3) is equivalent to

$$
\psi(0)+z \gamma^{2} \psi^{*}(0)=0
$$

with $z=-i$, see [15]. A computation shows that the condition (1.3) is conserved under the evolution of (1.1) and (1.2), and moreover, that if $\psi$ is of the form (1.3) then $\bar{\psi} \psi=0$. Consequently, under the assumption (1.3), the cubic Dirac equation (1.1) and the Dirac-Klein-Gordon system (1.3) reduce to equations which are linear in $\psi$. In particular, the argument of Chadam-Glassey gives scattering and global well-posedness for (1.1) and (1.2) for a class of large data [10. The structural condition (1.3) considered by Chadam and Glassey was introduced in the physics literature long before by Majorana [14 to describe fermions which are their own anti-particles, see 12 for an overview.

Our main Theorems 1.1 and 1.2 below pertain to solutions emanating from initial data which approximately satisfy the algebraic condition (1.4) with $|z|=1$. For the results concerning the cubic Dirac equation (1.1), we rely on the estimates obtain in [3, 4, 17. On the other hand, in the case of the Dirac-Klein-Gordon system (1.2), we require more refined estimates than those used in $[9$ to obtain the current sharpest small data global theory. The reason is that we have to deal with a large potential in the Dirac equation, which essentially is a free Klein-Gordon wave. Instead, we use refined estimates obtained in [8] which give a small power of a space-time $L_{t, x}^{4}$ norm on the righthand side.

The main result regarding the cubic Dirac equation is the following.

Theorem 1.1. Let $z \in \mathbb{C},|z|=1$, and $M \geqslant 0$. For any $A \geqslant 1$ there exists $\epsilon=\epsilon(A)>0$ such that for all initial data satisfying

$$
\|\psi(0)\|_{H^{1}\left(\mathbb{R}^{3}\right)} \leqslant A \text { and }\left\|\psi(0)+z \gamma^{2} \psi^{*}(0)\right\|_{H^{1}\left(\mathbb{R}^{3}\right)} \leqslant \epsilon,
$$

the cubic Dirac equation (1.1) is globally well-posed and solutions scatter to free solutions as $t \rightarrow \pm \infty$.

To be more precise, we prove Theorem 3.1 on a reduced system instead, which is equivalent for smooth solutions. In Theorem 1.1 we are forced to take $\epsilon$ much smaller than $A^{-1}$. The regularity assumption in Theorem 1.1 is sharp, in the sense that $\dot{H}^{1}\left(\mathbb{R}^{3}\right)$ is the scale invariant space. In particular, the regularity assumptions match the optimal results known in the small data case 3, 7. The importance of Theorem 1.1 is that we can take $A$ to be large, in particular, we obtain scattering for an open set of large data with essentially sharp regularity assumptions. Under stronger decay and regularity conditions, such results have been proven by Bachelot in [2]. Very recently, a similar result has been derived in [11] in the presence of a time independent potential and for initial data in $H^{1}\left(\mathbb{R}^{3}\right)$ with additional angular regularity.

We also have the corresponding version for the Dirac-Klein-Gordon system. Let $H_{\sigma}^{s}\left(\mathbb{R}^{3}\right)=\left(1-\Delta_{\mathbb{S}^{2}}\right)^{-\frac{\sigma}{2}} H^{s}\left(\mathbb{R}^{3}\right)$ be the subspace of the standard Sobolev space $H^{s}\left(\mathbb{R}^{3}\right)$ containing functions with $\sigma$ angular derivatives in $H^{s}\left(\mathbb{R}^{3}\right)$, equipped with the norm

$$
\|f\|_{H_{\sigma}^{s}}=\left\|\left(1-\Delta_{\mathbb{S}^{2}}\right)^{\frac{\sigma}{2}} f\right\|_{H^{s}}
$$


see [9, 8] for details. Note that $H^{s}\left(\mathbb{R}^{3}\right)=H_{0}^{s}\left(\mathbb{R}^{3}\right)$.

Theorem 1.2. Let $z \in \mathbb{C},|z|=1$. Suppose that either $s>0=\sigma$ and $2 M>m>0$, or $\sigma>0=s$ and $M, m>0$. For any $A \geqslant 1$, there exists $\epsilon=\epsilon(A)>0$, such that if

$$
\|\psi(0)\|_{H_{\sigma}^{s}\left(\mathbb{R}^{3}\right)} \leqslant A, \quad\|\phi(0)\|_{H_{\sigma}^{\frac{1}{2}+s}\left(\mathbb{R}^{3}\right)} \leqslant A, \quad\left\|\partial_{t} \phi(0)\right\|_{H_{\sigma}^{-\frac{1}{2}+s}\left(\mathbb{R}^{3}\right)} \leqslant A,
$$

and

$$
\left\|\psi(0)+z \gamma^{2} \psi^{*}(0)\right\|_{H_{\sigma}^{s}\left(\mathbb{R}^{3}\right)} \leqslant \epsilon
$$

then the system (1.2) is globally well-posed and solutions scatter to free solutions as $t \rightarrow \pm \infty$.

As for the cubic Dirac equation, we prove Theorem 4.1 on a reduced system instead, which is equivalent for smooth solutions.

We obtain an upper bound for $\epsilon$ which is the inverse exponential of a power of $A$, see Theorem 4.1 for more details. The Chadam-Glassey result in [10] corresponds to the case $z=i$ and $\epsilon=0$ (with additional smoothness assumptions on the data). A result similar to Theorem 1.2 under strong decay and regularity conditions has been established in [1. Notice that the small data results in [3, 7, 9] correspond to Theorems 1.1 and 1.2 respectively, in the case where $A$ is very small, since it clearly implies the condition on $\psi(0)+z \gamma^{2} \psi^{*}(0)$. Notice that $s=0$ is the critical regularity for (1.2).

Organisation of the paper. In Section 2 we perform an initial reduction which decouples the small and the large parts of the spinors. In Section 3 we reformulate and prove the main results concerning the Soler model. In Section 4 we reformulate and prove the main results on the Dirac-Klein-Gordon system.

\section{INITIAL REDUCTIONS}

Suppose we have data $\psi(0)$ satisfying the assumptions of Theorem 1.1 One way to proceed would be to linearise around the Chadam-Glassey type solutions. Thus decomposing

$$
\psi(0)=\psi_{N}(0)+\psi_{L}(0)
$$

where $\left\|\psi_{N}(0)\right\|_{H^{1}} \leqslant \epsilon$ and $\psi_{L}(0)+z \gamma^{2} \psi_{L}^{*}(0)=0$. Let $\psi_{L}$ denote the solution to the linear Dirac equation with data $\psi_{L}(0)$. As mentioned in the introduction, for all times we have $\bar{\psi}_{L} \psi_{L}=0$. Consequently, the remaining term $\psi_{N}=\psi-\psi_{L}$ satisfies the equation

$$
-i \gamma^{\mu} \partial_{\mu} \psi_{N}+M \psi_{N}=\left(\bar{\psi}_{L} \psi_{N}+\bar{\psi}_{N} \psi_{L}\right) \psi+\bar{\psi}_{N} \psi_{N} \psi
$$

The last term is small since $\psi_{N}(0)$ is small. On the other hand, it is not at all clear that the first term $\left(\bar{\psi}_{L} \psi_{N}+\bar{\psi}_{N} \psi_{L}\right) \psi$ should be small, since it contains terms of the schematic form $\psi_{L}^{2} \psi_{N}$, and $\psi_{L}$ can be large. In particular, if we wanted to use the linearised equation to prove Theorem 1.1 we would be forced to absorb these terms into the left hand side, which would significantly complicate the required multilinear estimates. It turns out that there is a better way to decompose $\psi$, which avoids this problem. In particular, we can exploit the multilinear estimates already contained in [3, 7. A similar comment applies to the proof for the DiracKlein-Gordon system, Theorem 1.2 However, a significant additional difficulty arises in the case where the data for $\phi$ is large.

We start with the following observation, see [14, 13, 10, we follow [15]. 
Lemma 2.1. Assume that $\psi$ is a classical solution of

$$
-i \gamma^{\mu} \partial_{\mu} \psi+M \psi=V \psi
$$

for some real-valued, scalar, and locally integrable function $V: \mathbb{R}^{1+3} \rightarrow \mathbb{R}$. Then for any $z \in \mathbb{C}$ we have

$$
\left\|\psi(t)+z \gamma^{2} \psi^{*}(t)\right\|_{L_{x}^{2}}=\left\|\psi(0)+z \gamma^{2} \psi^{*}(0)\right\|_{L_{x}^{2}} .
$$

Proof. A computation shows that $\gamma^{\mu} \gamma^{2}=-\gamma^{2}\left(\gamma^{\mu}\right)^{*}$ which implies that

$-i \gamma^{\mu} \partial_{\mu}\left(\psi+z \gamma^{2} \psi^{*}\right)=-i \gamma^{\mu} \partial_{\mu} \psi+z \gamma^{2}\left(-i \gamma^{\mu} \partial_{\mu} \psi\right)^{*}=-M\left(\psi+z \gamma^{2} \psi^{*}\right)+V\left(\psi+z \gamma^{2} \psi^{*}\right)$.

Result now follows by multiplying by $i\left(\psi+z \gamma^{2} \psi^{*}\right)^{\dagger} \gamma^{0}$, taking the real part, and then integrating over $\mathbb{R}^{3}$.

We can now rewrite the cubic Dirac equation (1.1). Let $\varphi, \chi: \mathbb{R}^{1+3} \rightarrow \mathbb{C}^{4}$ be smooth enough and solve

$$
\begin{aligned}
& -i \gamma^{\mu} \partial_{\mu} \varphi+M \varphi=(\bar{\varphi} \chi+\bar{\chi} \varphi) \varphi \\
& -i \gamma^{\mu} \partial_{\mu} \chi+M \chi=(\bar{\varphi} \chi+\bar{\chi} \varphi) \chi
\end{aligned}
$$

with data

$$
\varphi(0)=\frac{1}{2}\left(\psi(0)+z \gamma^{2} \psi^{*}(0)\right), \quad \chi(0)=\frac{1}{2}\left(\psi(0)-z \gamma^{2} \psi^{*}(0)\right) .
$$

Then a computation using Lemma 2.1 implies that for all $t \in \mathbb{R}$ and $|z|=1$ we have

$$
\varphi(t)+z \gamma^{2} \varphi^{*}(t)=0, \quad \chi(t)-z \gamma^{2} \chi^{*}(t)=0
$$

and moreover that $\bar{\varphi} \varphi=\bar{\chi} \chi=0$. Consequently, if we let $\psi=\varphi+\chi$, we obtain a solution to the cubic Dirac equation (1.1). Similarly, in the case of the Dirac-KleinGordon system (1.2), let $\varphi, \chi: \mathbb{R}^{1+3} \rightarrow \mathbb{C}^{4}$ and $\phi: \mathbb{R}^{1+3} \rightarrow \mathbb{R}$ be smooth enough and solve

$$
\begin{aligned}
-i \gamma^{\mu} \partial_{\mu} \varphi+M \varphi & =\phi \varphi \\
-i \gamma^{\mu} \partial_{\mu} \chi+M \chi & =\phi \chi \\
\square \phi+m^{2} \phi & =\bar{\varphi} \chi+\bar{\chi} \varphi
\end{aligned}
$$

with data

$$
\varphi(0)=\frac{1}{2}\left(\psi(0)+z \gamma^{2} \psi^{*}(0)\right), \quad \chi(0)=\frac{1}{2}\left(\psi(0)-z \gamma^{2} \psi^{*}(0)\right) .
$$

As in the case of the cubic Dirac equation, an application of Lemma 2.1 implies that

$$
\varphi(t)+z \gamma^{2} \varphi^{*}(t)=0, \quad \chi(t)-z \gamma^{2} \chi^{*}(t)=0
$$

and hence provided $|z|=1$ we have $\bar{\varphi} \varphi=\bar{\chi} \chi=0$. Consequently, letting $\psi=\varphi+\chi$ we get a solution to (1.2). For technical reasons, we prefer to work with a first order system. Defining $\phi_{+}=\phi+i\langle\nabla\rangle^{-1} \partial_{t} \phi$, as $\phi$ is real-valued, we obtain

$$
\begin{aligned}
-i \gamma^{\mu} \partial_{\mu} \varphi+M \varphi & =\operatorname{Re}\left(\phi_{+}\right) \varphi \\
-i \gamma^{\mu} \partial_{\mu} \chi+M \chi & =\operatorname{Re}\left(\phi_{+}\right) \chi \\
-i \partial_{t} \phi_{+}+\langle\nabla\rangle_{m} \phi_{+} & =\langle\nabla\rangle_{m}^{-1}(\bar{\varphi} \chi+\bar{\chi} \varphi)
\end{aligned}
$$


with data

$$
\begin{aligned}
& \qquad(0)=\frac{1}{2}\left(\psi(0)+z \gamma^{2} \psi^{*}(0)\right), \quad \chi(0)=\frac{1}{2}\left(\psi(0)-z \gamma^{2} \psi^{*}(0)\right), \\
& \text { and } \phi_{+}(0)=\phi(0)+i\langle\nabla\rangle^{-1} \partial_{t} \phi(0) .
\end{aligned}
$$

Conversely, from $\phi_{+}$we can recover $\phi$ by taking the real part of $\phi_{+}$.

\section{Cubic Dirac equation}

We begin by introducing some notation. Let $\Pi_{ \pm}$be the projection

$$
\Pi_{ \pm}=\frac{1}{2}\left(I \pm\langle\nabla\rangle_{M}^{-1}\left(-i \gamma^{0} \gamma^{j} \partial_{j}+M \gamma^{0}\right)\right)
$$

let $\mathcal{U}_{m}^{ \pm}(t)=e^{\mp i t\langle\nabla\rangle_{m}}$ be the propagator for the homogeneous half-wave equation, let

$$
\mathcal{U}_{M}(t)=\mathcal{U}_{M}^{+}(t) \Pi_{+}+\mathcal{U}_{M}^{-}(t) \Pi_{-}
$$

be the propagator for the homogeneous Dirac equation, and let

$$
\begin{aligned}
\mathcal{I}_{t_{0}}^{ \pm, m}(F)(t) & =i \int_{t_{0}}^{t} \mathcal{U}_{m}^{ \pm}\left(t-t_{0}-t^{\prime}\right) F\left(t^{\prime}\right) d t^{\prime}, \\
\mathcal{I}_{t_{0}}^{M}(G)(t) & =i \int_{t_{0}}^{t} \mathcal{U}_{M}\left(t-t_{0}-t^{\prime}\right) \gamma^{0} G\left(t^{\prime}\right) d t^{\prime} .
\end{aligned}
$$

be the corresponding Duhamel integrals.

The previous section implies that for smooth solutions (1.1) and (2.1) are equivalent, so that we focus on proving the following.

Theorem 3.1. Let $z \in \mathbb{C},|z|=1$, and $M \geqslant 0$. There exists $c \in(0,1)$, such that for any $A>0$ and $\epsilon \leqslant c A^{-1}$, if the initial data satisfy

$$
\|\varphi(0)\|_{H^{1}} \leqslant \epsilon, \quad\|\chi(0)\|_{H^{1}} \leqslant A,
$$

then (2.1) is globally well-posed and the solutions scatter in $H^{1}\left(\mathbb{R}^{3}\right)$ to free solutions as $t \rightarrow \pm \infty$, i.e. there exist $\varphi_{ \pm \infty} \in H^{1}\left(\mathbb{R}^{3}\right)$ and $\chi_{ \pm \infty} \in H^{1}\left(\mathbb{R}^{3}\right)$, such that

$$
\lim _{t \rightarrow \pm \infty}\left\|\varphi(t)-\mathcal{U}_{M}(t) \varphi_{ \pm \infty}\right\|_{H^{1}}=0 \text { and } \lim _{t \rightarrow \pm \infty}\left\|\chi(t)-\mathcal{U}_{M}(t) \chi_{ \pm \infty}\right\|_{H^{1}}=0 .
$$

Proof. Let $X \subset C\left(\mathbb{R}, H^{1}\left(\mathbb{R}^{3}\right)\right)$ be the Banach space constructed in [3] in the massive case $(M>0)$ and in [7] in the massless case $(M=0)$. Further, let $\|\cdot\|_{X}$ denote the norm obtained by multiplying by the norms from [3, [7 by a small enough constant, such that for all solutions $\varphi \in X$ to the inhomogeneous Dirac equation

$$
-i \gamma^{\mu} \partial_{\mu} \varphi+M \varphi=\left(\overline{\varphi^{(1)}} \varphi^{(2)}\right) \varphi^{(3)}
$$

the bound

$$
\|\varphi\|_{X} \leqslant\|\varphi(0)\|_{H^{1}\left(\mathbb{R}^{3}\right)}+C\left\|\varphi^{(1)}\right\|_{X}\left\|\varphi^{(2)}\right\|_{X}\left\|\varphi^{(3)}\right\|_{X}
$$

holds. Consider the set

$$
\mathcal{X}=\left\{(\varphi, \chi) \in X \times X \mid\|\varphi\|_{X} \leqslant 2\|\varphi(0)\|_{H^{1}},\|\chi\|_{X} \leqslant 2\|\chi(0)\|_{H^{1}}\right\}
$$

and, for $A, \epsilon>0$, the norm

$$
\|(\varphi, \chi)\|_{\mathcal{X}}=\epsilon^{-1}\|\varphi\|_{X}+A^{-1}\|\chi\|_{X} .
$$


$\mathcal{X}$ is a complete metric space. Let $\mathcal{T}=\left(T_{1}, T_{2}\right)$ denote the standard (inhomogeneous) solution map for (2.1) constructed from the Duhamel formula. The bound (3.1) together with the assumption on the initial data show that if $(\varphi, \chi) \in \mathcal{X}$ then

$$
\begin{aligned}
\left\|T_{1}(\varphi, \chi)\right\|_{X} & \leqslant\|\varphi(0)\|_{H^{1}}+2 C\|\varphi\|_{X}^{2}\|\chi\|_{X} \leqslant\|\varphi(0)\|_{H^{1}}+2^{4} C\|\varphi(0)\|_{H^{1}}^{2}\|\chi(0)\|_{H^{1}} \\
& \leqslant\left(1+2^{4} C A \epsilon\right)\|\varphi(0)\|_{H^{1}}
\end{aligned}
$$

and similarly

$$
\left\|T_{2}(\varphi, \chi)\right\|_{X} \leqslant\|\chi(0)\|_{H^{1}}+2 C\|\chi\|_{X}^{2}\|\varphi\|_{X} \leqslant\left(1+2^{4} C A \epsilon\right)\|\chi(0)\|_{H^{1}} .
$$

Consequently, provided that $\epsilon \leq\left(2^{4} C A\right)^{-1}$, we see that $\mathcal{T}: \mathcal{X} \rightarrow \mathcal{X}$. Next, we verify that $\mathcal{T}$ is a contraction. For $\left(\varphi_{1}, \chi_{1}\right),\left(\varphi_{2}, \chi_{2}\right) \in \mathcal{X}$ another application of (3.1) gives

$$
\left\|T_{1}\left(\varphi_{1}, \chi_{1}\right)-T_{1}\left(\varphi_{2}, \chi_{2}\right)\right\|_{X} \leqslant 2^{4} C A \epsilon\left\|\varphi_{1}-\varphi_{2}\right\|_{X}+2^{3} C \epsilon^{2}\left\|\chi_{1}-\chi_{2}\right\|_{X},
$$

and similarly

$$
\left\|T_{2}\left(\varphi_{1}, \chi_{1}\right)-T_{2}\left(\varphi_{2}, \chi_{2}\right)\right\|_{X} \leqslant 2^{4} C A \epsilon\left\|\chi_{1}-\chi_{2}\right\|_{X}+2^{3} C A^{2}\left\|\varphi_{1}-\varphi_{2}\right\|_{X} .
$$

This implies

$$
\left\|\mathcal{T}\left(\varphi_{1}, \chi_{1}\right)-\mathcal{T}\left(\varphi_{2}, \chi_{2}\right)\right\|_{\mathcal{X}} \leqslant 2^{6} C A \epsilon\left\|\left(\varphi_{1}, \chi_{1}\right)-\left(\varphi_{2}, \chi_{2}\right)\right\|_{\mathcal{X}}
$$

Therefore, choosing $\epsilon \leq\left(2^{7} C A\right)^{-1}$, the map $\mathcal{T}: \mathcal{X} \rightarrow \mathcal{X}$ is a contraction with respect to $\|\cdot\|_{\mathcal{X}}$, hence it has a unique fixed point in $\mathcal{X}$, and standard arguments show the continuity of the flow map. The scattering claim follows from the finiteness of both $\|\varphi\|_{X}$ and $\|\chi\|_{X}$, because this implies that the pull-backs of $\varphi$ and $\chi$ along the free evolution, as maps from $\mathbb{R}$ to $H^{1}\left(\mathbb{R}^{3}\right)$, have finite quadratic variation, see [3, 7] for the details.

\section{The Dirac-KLein-Gordon system}

Let $P_{\lambda}$ be the standard Littlewood-Paley projections onto dyadic frequencies of size $\lambda$, and take $H_{N}$ to be the projection onto angular frequencies of size $N$, see 8 , Section 2] for precise definitions. If $s \geqslant 0$ and $\sigma=0$, we define

$$
\|f\|_{\mathbf{D}_{0}^{s}(I)}=\left\|\langle\nabla\rangle^{s} f\right\|_{L^{4}\left(I \times \mathbb{R}^{3}\right)} .
$$

On the other hand, for $s \geqslant 0$ and $\sigma>0$, we take

$$
\|f\|_{\mathbf{D}_{\sigma}^{s}(I)}=\left(\sum_{N \geqslant 1} N^{2 \sigma}\left\|\langle\nabla\rangle^{s} H_{N} f\right\|_{L^{4}\left(I \times \mathbb{R}^{3}\right)}^{2}\right)^{\frac{1}{2}} .
$$

The results in Section 2 imply that for smooth solutions (1.2) and (2.3) are equivalent, so that we focus on proving the following.

Theorem 4.1. Let $z \in \mathbb{C},|z|=1$. Suppose that either $s>0=\sigma$ and $2 M>m>0$, or $\sigma>0=s$ and $M, m>0$. There exist $0<c<1$ and $\gamma>1$, such that for any $A \geqslant 1$ and any $\epsilon \leqslant c \exp \left(-A^{\gamma}\right)$, if

$$
\|\varphi(0)\|_{H_{\sigma}^{s}\left(\mathbb{R}^{3}\right)} \leqslant \epsilon, \quad\|\chi(0)\|_{H_{\sigma}^{s}\left(\mathbb{R}^{3}\right)} \leqslant A, \quad\left\|\phi_{+}(0)\right\|_{H_{\sigma}^{\frac{1}{2}+s}\left(\mathbb{R}^{3}\right)} \leqslant A,
$$


then the system (2.3) is globally well-posed and scatters to free solutions as $t \rightarrow \pm \infty$, i.e. there exist $\varphi_{ \pm \infty} \in H_{\sigma}^{s}\left(\mathbb{R}^{3}\right), \chi_{ \pm \infty} \in H_{\sigma}^{s}\left(\mathbb{R}^{3}\right)$ and $\phi_{ \pm \infty} \in H_{\sigma}^{\frac{1}{2}+s}\left(\mathbb{R}^{3}\right)$, such that

$$
\begin{aligned}
& \lim _{t \rightarrow \pm \infty}\left\|\varphi(t)-\mathcal{U}_{M}(t) \varphi_{ \pm \infty}\right\|_{H_{\sigma}^{s}}=0, \lim _{t \rightarrow \pm \infty}\left\|\chi(t)-\mathcal{U}_{M}(t) \chi_{ \pm \infty}\right\|_{H_{\sigma}^{s}}=0, \\
& \text { and } \lim _{t \rightarrow \pm \infty}\left\|\phi_{+}(t)-\mathcal{U}_{m}^{+}(t) \phi_{ \pm \infty}\right\|_{H_{\sigma}^{s+\frac{1}{2}}}=0 .
\end{aligned}
$$

Before we turn to its proof, we summarise the results we require from 8 .

Lemma 4.2. Let $s, \sigma \in \mathbb{R}$, and $I$ be any interval of the form $I=\left[t_{1}, t_{2}\right),-\infty<$ $t_{1}<t_{2} \leq \infty$. There exist Banach function spaces $\mathbf{F}_{M}^{s, \sigma}(I)$ and $\mathbf{V}_{+, m}^{s, \sigma}(I)$ and $C_{0} \geqslant 1$ with the following properties:

(i) $C_{0}^{\infty}\left(I \times \mathbb{R}^{3} ; \mathbb{C}^{4}\right) \subset \mathbf{F}_{M}^{s, \sigma}(I), C_{0}^{\infty}\left(I \times \mathbb{R}^{3} ; \mathbb{C}\right) \subset \mathbf{V}_{+, m}^{s, \sigma}(I)$, and

$$
\mathbf{F}_{M}^{s, \sigma}(I) \hookrightarrow C_{b}\left(I ; H_{\sigma}^{s}\left(\mathbb{R}^{3} ; \mathbb{C}^{4}\right)\right), \quad \mathbf{V}_{+, m}^{s, \sigma}(I) \hookrightarrow C_{b}\left(I ; H_{\sigma}^{s}\left(\mathbb{R}^{3} ; \mathbb{C}\right)\right) .
$$

(ii) For $\psi \in \mathbf{F}_{M}^{s, \sigma}(I), \phi_{+} \in \mathbf{V}_{+, m}^{s, \sigma}(I)$, and for any $I^{\prime}=\left[s_{1}, s_{2}\right) \subset I$, we have $\left.\psi\right|_{I^{\prime}} \in \mathbf{F}_{M}^{s, \sigma}\left(I^{\prime}\right),\left.\phi_{+}\right|_{I^{\prime}} \in \mathbf{V}_{+, m}^{s, \sigma}\left(I^{\prime}\right)$, and

$$
\left\|\left.\psi\right|_{I^{\prime}}\right\|_{\mathbf{F}_{M}^{s, \sigma}\left(I^{\prime}\right)} \leqslant C_{0}\|\psi\|_{\mathbf{F}_{M}^{s, \sigma}(I)}, \quad\left\|\left.\phi_{+}\right|_{I^{\prime}}\right\|_{\mathbf{V}_{+, m}^{s+\frac{1}{2}, \sigma}\left(I^{\prime}\right)} \leqslant C_{0}\|\phi\|_{\mathbf{V}_{+, m}^{s+\frac{1}{2}, \sigma}(I)} .
$$

(iii) For $\psi_{0} \in H_{\sigma}^{s}\left(\mathbb{R}^{3} ; \mathbb{C}^{4}\right)$ and $\phi_{0} \in H_{\sigma}^{s}\left(\mathbb{R}^{3} ; \mathbb{C}\right)$ we have $\mathcal{U}_{M}(t) \psi_{0} \in \mathbf{F}_{M}^{s, \sigma}(I)$, $\mathcal{U}_{m}^{+}(t) \phi_{0} \in \mathbf{V}_{+, m}^{s, \sigma}(I)$, and the bounds

$$
\left\|\mathcal{U}_{M} \psi_{0}\right\|_{\mathbf{F}_{M}^{s, \sigma}(I)} \leqslant\left\|\psi_{0}\right\|_{H_{\sigma}^{s}}, \quad\left\|\mathcal{U}_{m}^{+} \phi_{0}\right\|_{\mathbf{V}_{+, m}^{s, \sigma}(I)} \leqslant\left\|\phi_{0}\right\|_{H_{\sigma}^{s}} .
$$

(iv) For $\psi \in \mathbf{F}_{M}^{s, \sigma}\left(\left[t_{1}, t_{2}\right)\right)$ and $\phi_{+} \in \mathbf{V}_{+, m}^{s, \sigma}\left(\left[t_{1}, t_{2}\right)\right)$ the limits

$\lim _{t \rightarrow t_{2}} \mathcal{U}_{M}(-t) \psi(t) \in H^{s}\left(\mathbb{R}^{3} ; \mathbb{C}^{4}\right)$ and $\lim _{t \rightarrow t_{2}} \mathcal{U}_{m}^{+}(-t) \phi_{+}(t) \in H^{s}\left(\mathbb{R}^{3} ; \mathbb{C}\right)$

exist.

(v) For $\phi_{+} \in \mathbf{V}_{+, m}^{s+\frac{1}{2}, \sigma}(I)$ we have the Strichartz-type estimate

$$
\left\|\phi_{+}\right\|_{\mathbf{D}_{\sigma}^{s(I)}} \leqslant C_{0}\left\|\phi_{+}\right\|_{\mathbf{V}_{+, m}^{s+\frac{1}{2}, \sigma}(I)} .
$$

(vi) Suppose that either $s>0=\sigma$ and $2 M>m>0$, or $\sigma>0=s$ and $M, m>0$. There exists $\theta \in(0,1)$, such that for any $t_{0} \in I$ the Duhamel operators

$$
\begin{aligned}
\mathbf{V}_{+, m}^{s+\frac{1}{2}, \sigma}(I) \times \mathbf{F}_{M}^{s, \sigma}(I) \ni\left(\phi_{+}, \varphi\right) & \mapsto \mathcal{I}_{t_{0}}^{M}\left(\operatorname{Re}\left(\phi_{+}\right) \varphi\right) \in \mathbf{F}_{M}^{s, \sigma}(I) \\
\mathbf{F}_{M}^{s, \sigma}(I) \times \mathbf{F}_{M}^{s, \sigma}(I) \ni(\chi, \varphi) & \mapsto \mathcal{I}_{t_{0}}^{+, m}\left(\langle\nabla\rangle_{m}^{-1}(\bar{\chi} \varphi)\right) \in \mathbf{V}_{+, m}^{s+\frac{1}{2}, \sigma}(I)
\end{aligned}
$$

are well-defined and the following estimates hold:

$$
\begin{aligned}
\left\|\mathcal{I}_{t_{0}}^{M}\left(\operatorname{Re}\left(\phi_{+}\right) \varphi\right)\right\|_{\mathbf{F}_{M}^{s, \sigma}(I)} & \leqslant C_{0}\left\|\phi_{+}\right\|_{\mathbf{D}_{\sigma}^{s}(I)}^{\theta}\left\|\phi_{+}\right\|_{\mathbf{V}_{+, m}^{s+\frac{1}{2}, \sigma}(I)}^{1-\theta}\|\varphi\|_{\mathbf{F}_{M}^{s, \sigma}(I)}, \\
\left\|\mathcal{I}_{t_{0}}^{+, m}\left(\langle\nabla\rangle_{m}^{-1}(\bar{\chi} \varphi)\right)\right\|_{\mathbf{V}_{+, m}^{s+\frac{1}{2}, \sigma}(I)} & \leqslant C_{0}\|\chi\|_{\mathbf{F}_{M}^{s, \sigma}(I)}\|\varphi\|_{\mathbf{F}_{M}^{s, \sigma}(I) .} .
\end{aligned}
$$

Proof. For details see Section 2, Lemma 2.1, and Theorem 3.2 in [8].

The first step in the proof of Theorem 4.1, is to prove the following local result. 
Theorem 4.3. Suppose that either $s>0=\sigma$ and $2 M>m>0$, or $\sigma>0=s$ and $M, m>0$. There exist $\theta, c \in(0,1)$ and $C>1$, such that for any $A, B \geqslant 1$ and any $0<\alpha \leqslant c A^{-1}$ and $0<\beta \leqslant c B^{\frac{\theta-1}{\theta}}$, and for any interval $I=\left[t_{1}, t_{2}\right) \subset \mathbb{R}$ and $t_{0} \in I$, if we have

$$
\left\|\varphi_{0}\right\|_{H_{\sigma}^{s}\left(\mathbb{R}^{3}\right)} \leqslant \alpha, \quad\left\|\chi_{0}\right\|_{H_{\sigma}^{s}\left(\mathbb{R}^{3}\right)} \leqslant A,
$$

and

$$
\left\|\mathcal{U}_{m}^{+}\left(\cdot-t_{0}\right) \phi_{0}\right\|_{\mathbf{D}_{\sigma}^{s}(I)} \leqslant \beta, \quad\left\|\phi_{0}\right\|_{H_{\sigma}^{\frac{1}{2}+s}\left(\mathbb{R}^{3}\right)} \leqslant B
$$

then there exists a unique solution $\left(\varphi, \chi, \phi_{+}\right) \in \mathbf{F}_{M}^{s, \sigma}(I) \times \mathbf{F}_{M}^{s, \sigma}(I) \times \mathbf{V}_{+, m}^{s+\frac{1}{2}, \sigma}(I)$ of (2.3) on $I \times \mathbb{R}^{3}$ with initial condition $\left(\varphi, \chi, \phi_{+}\right)\left(t_{0}\right)=\left(\varphi_{0}, \chi_{0}, \phi_{0}\right)$. Moreover the solution depends continuously on the initial data and satisfies the bounds

$$
\begin{aligned}
& \sup _{t \in I}\|\varphi(t)\|_{H_{\sigma}^{s}\left(\mathbb{R}^{3}\right)} \leqslant 2\left\|\varphi_{0}\right\|_{H_{\sigma}^{s}\left(\mathbb{R}^{3}\right)}, \quad \sup _{t \in I}\|\chi(t)\|_{H_{\sigma}^{s}\left(\mathbb{R}^{3}\right)} \leqslant 2\left\|\chi_{0}\right\|_{H_{\sigma}^{s}\left(\mathbb{R}^{3}\right)}, \\
& \sup _{t \in I}\left\|\phi_{+}(t)-\mathcal{U}_{m}^{+}\left(t-t_{0}\right) \phi_{0}\left(t_{0}\right)\right\|_{H_{\sigma}^{\frac{1}{2}+s}\left(\mathbb{R}^{3}\right)} \leqslant C\left\|\varphi_{0}\right\|_{H_{\sigma}^{s}\left(\mathbb{R}^{3}\right)}\left\|\chi_{0}\right\|_{H_{\sigma}^{s}\left(\mathbb{R}^{3}\right) .}
\end{aligned}
$$

Proof. For convenience, let $\varphi_{L}(t)=\mathcal{U}_{M}\left(t-t_{0}\right) \varphi_{0}, \chi_{L}(t)=\mathcal{U}_{M}\left(t-t_{0}\right) \chi_{0}$, and $\phi_{+, L}(t)=\mathcal{U}_{m}^{+}\left(t-t_{0}\right) \phi_{0}$. Let $C_{0} \geqslant 1$ and $\theta \in(0,1)$ be as in Lemma 4.2. Define $S$ as the set of all $\left(\varphi, \chi, \phi_{+}\right) \in \mathbf{F}_{M}^{s, \sigma}(I) \times \mathbf{F}_{M}^{s, \sigma}(I) \times \mathbf{V}_{+, m}^{s+\frac{1}{2}, \sigma}(I)$ satisfying

$$
\begin{aligned}
\left\|\varphi-\varphi_{L}\right\|_{\mathbf{F}_{M}^{s, \sigma}(I)} & \leqslant\left\|\varphi_{0}\right\|_{H_{\sigma}^{s}}, \quad\left\|\chi-\chi_{L}\right\|_{\mathbf{F}_{M}^{s, \sigma}(I)} \leqslant\left\|\chi_{0}\right\|_{H_{\sigma}^{s}}, \\
\left\|\phi_{+}-\phi_{+, L}\right\|_{\mathbf{v}_{+, m}^{s+\frac{1}{2}, \sigma}(I)} & \leqslant 2^{3} C_{0}\left\|\varphi_{0}\right\|_{H_{\sigma}^{s}}\left\|\chi_{0}\right\|_{H_{\sigma}^{s}} .
\end{aligned}
$$

It is a complete metric space with respect to the norm

$$
\left\|\left(\varphi, \chi, \phi_{+}\right)\right\|_{S}:=\alpha^{-1}\|\varphi\|_{\mathbf{F}_{M}^{s, \sigma}(I)}+A^{-1}\|\chi\|_{\mathbf{F}_{M}^{s, \sigma}(I)}+\eta^{-1}\left\|\phi_{+}\right\|_{\mathbf{V}_{+, m}^{s+\frac{1}{2}, \sigma}(I)},
$$

where $\eta>0$ will be chosen later. Let

$\mathcal{T}=\left(T_{1}, T_{2}, T_{3}\right): \mathbf{F}_{M}^{s, \sigma}(I) \times \mathbf{F}_{M}^{s, \sigma}(I) \times \mathbf{V}_{+, m}^{s+\frac{1}{2}, \sigma}(I) \rightarrow \mathbf{F}_{M}^{s, \sigma}(I) \times \mathbf{F}_{M}^{s, \sigma}(I) \times \mathbf{V}_{+, m}^{s+\frac{1}{2}, \sigma}(I)$ be defined as

$$
\mathcal{T}\left(\varphi, \chi, \phi_{+}\right)=\left(\begin{array}{c}
\mathcal{U}_{M}\left(\cdot-t_{0}\right) \varphi_{0}+\mathcal{I}_{t_{0}}^{M}\left(\operatorname{Re}\left(\phi_{+}\right) \varphi\right) \\
\mathcal{U}_{M}\left(\cdot-t_{0}\right) \chi_{0}+\mathcal{I}_{t_{0}}^{M}\left(\operatorname{Re}\left(\phi_{+}\right) \chi\right) \\
\mathcal{U}_{m}^{+}\left(\cdot-t_{0}\right) \phi_{+, 0}+\mathcal{I}_{t_{0}}^{+, m}\left(\langle\nabla\rangle_{m}^{-1}(\bar{\varphi} \chi+\bar{\chi} \varphi)\right)
\end{array}\right),
$$

see Lemma 4.2. Fixed points of $\mathcal{T}$ are solutions of (2.3) with the given data at time $t_{0}$. For $\left(\varphi, \chi, \phi_{+}\right) \in S$ we infer that

$$
\begin{aligned}
& \|\varphi\|_{\mathbf{F}_{M}^{s, \sigma}(I)} \leqslant\left\|\varphi-\varphi_{L}\right\|_{\mathbf{F}_{M}^{s, \sigma}(I)}+\left\|\varphi_{L}\right\|_{\mathbf{F}_{M}^{s, \sigma}(I)} \leqslant 2\left\|\varphi_{0}\right\|_{H_{\sigma}^{s}} \leqslant 2 \alpha, \\
& \|\chi\|_{\mathbf{F}_{M}^{s, \sigma}(I)} \leqslant\left\|\chi-\chi_{L}\right\|_{\mathbf{F}_{M}^{s, \sigma}(I)}+\left\|\chi_{L}\right\|_{\mathbf{F}_{M}^{s, \sigma}(I)} \leqslant 2\left\|\chi_{0}\right\|_{H_{\sigma}^{s}} \leqslant 2 A,
\end{aligned}
$$

and similarly,

$$
\begin{gathered}
\left\|\phi_{+, L}\right\|_{\mathbf{D}_{\sigma}^{s}(I)}^{\theta}\left\|\phi_{+, L}\right\|_{\mathbf{V}_{+, m}^{s+\frac{1}{2}, \sigma}(I)}^{1-\theta} \leqslant \beta^{\theta} B^{1-\theta} \\
\left\|\phi_{+}-\phi_{+, L}\right\|_{\mathbf{D}_{\sigma}^{s}(I)}^{\theta}\left\|\phi_{+}-\phi_{+, L}\right\|_{\mathbf{V}_{+, m}^{s+\frac{1}{2}, \sigma}(I)}^{1-\theta} \leqslant 2^{3} C_{0}^{1+\theta}\left\|\varphi_{0}\right\|_{H_{\sigma}^{s}}\left\|\chi_{0}\right\|_{H_{\sigma}^{s}} \leqslant 2^{3} C_{0}^{2} \alpha A .
\end{gathered}
$$

If $\alpha \leqslant\left(2^{5} C_{0}^{3} A\right)^{-1}$ and $\beta \leqslant\left(4 C_{0} B^{1-\theta}\right)^{-\frac{1}{\theta}}$, Lemma 4.2 implies

$$
\left\|T_{1}\left(\varphi, \chi, \phi_{+}\right)-\varphi_{L}\right\|_{\mathbf{F}_{M}^{s, \sigma}(I)} \leqslant\left(2 C_{0} \beta^{\theta} B^{1-\theta}+2^{4} C_{0}^{3} \alpha A\right)\left\|\varphi_{0}\right\|_{H_{\sigma}^{s}} \leqslant\left\|\varphi_{0}\right\|_{H_{\sigma}^{s}},
$$


and

$$
\left\|T_{2}\left(\varphi, \chi, \phi_{+}\right)-\chi_{L}\right\|_{\mathbf{F}_{M}^{s, \sigma}(I)} \leqslant\left(2 C_{0} \beta^{\theta} B^{1-\theta}+2^{4} C_{0}^{3} \alpha A\right)\left\|\chi_{0}\right\|_{H_{\sigma}^{s}} \leqslant\left\|\chi_{0}\right\|_{H_{\sigma}^{s}}
$$

as well as

$$
\left\|T_{3}\left(\varphi, \chi, \phi_{+}\right)-\phi_{+, L}\right\|_{\mathbf{v}_{+, m}^{s+\frac{1}{2}, \sigma}(I)} \leqslant 2^{3} C_{0}\left\|\varphi_{0}\right\|_{H_{\sigma}^{s}}\left\|\chi_{0}\right\|_{H_{\sigma}^{s}} .
$$

We will now show that $\mathcal{T}: S \rightarrow S$ is a contraction, provided that $\alpha, \beta$ are chosen small enough. Let $\left(\varphi, \chi, \phi_{+}\right) \in S$ and $\left(\tilde{\varphi}, \tilde{\chi}, \tilde{\phi}_{+}\right) \in S$. Then, by Lemma 4.2 ,

$$
\begin{aligned}
\left\|T_{1}\left(\varphi, \chi, \phi_{+}\right)-T_{1}\left(\tilde{\varphi}, \tilde{\chi}, \tilde{\phi}_{+}\right)\right\|_{\mathbf{F}_{M}^{s, \sigma}(I)} \leqslant & \left(C_{0} \beta^{\theta} B^{1-\theta}+2^{3} C_{0}^{3} \alpha A\right)\|\varphi-\tilde{\varphi}\|_{\mathbf{F}_{M}^{s, \sigma}(I)} \\
& +2 C_{0}^{2} \alpha\left\|\phi_{+}-\tilde{\phi}_{+}\right\|_{\mathbf{v}_{+, m}^{s+\frac{1}{2}, \sigma}(I)}
\end{aligned}
$$

and

$$
\begin{aligned}
\left\|T_{2}\left(\varphi, \chi, \phi_{+}\right)-T_{2}\left(\tilde{\varphi}, \tilde{\chi}, \tilde{\phi}_{+}\right)\right\|_{\mathbf{F}_{M}^{s, \sigma}(I)} \leqslant & \left(C_{0} \beta^{\theta} B^{1-\theta}+2^{3} C_{0}^{3} \alpha A\right)\|\chi-\tilde{\chi}\|_{\mathbf{F}_{M}^{s, \sigma}(I)} \\
& +2 C_{0}^{2} A\left\|\phi_{+}-\tilde{\phi}_{+}\right\|_{\mathbf{v}_{+, m}^{s+\frac{1}{2}, \sigma}(I)},
\end{aligned}
$$

as well as

$$
\left\|T_{3}\left(\varphi, \chi, \phi_{+}\right)-T_{3}\left(\tilde{\varphi}, \tilde{\chi}, \tilde{\phi}_{+}\right)\right\|_{\mathbf{v}_{+, m}^{s+\frac{1}{2}, \sigma}(I)} \leqslant 2^{2} C_{0} \alpha\|\chi-\tilde{\chi}\|_{\mathbf{F}_{M}^{s, \sigma}(I)}+2^{2} C_{0} A\|\varphi-\tilde{\varphi}\|_{\mathbf{F}_{M}^{s, \sigma}(I)} .
$$

We obtain

$$
\begin{aligned}
\| \mathcal{T}\left(\varphi, \chi, \phi_{+}\right) & -\mathcal{T}\left(\tilde{\varphi}, \tilde{\chi}, \tilde{\phi}_{+}\right)\left\|_{S} \leqslant 4 C_{0}^{2} \eta \eta^{-1}\right\| \phi_{+}-\tilde{\phi}_{+} \|_{\mathbf{v}_{+, m}^{s+\frac{1}{2}, \sigma}(I)} \\
& +\left(C_{0} \beta^{\theta} B^{1-\theta}+2^{3} C_{0}^{3} \alpha A+2^{2} C_{0} A \alpha \eta^{-1}\right) \alpha^{-1}\|\varphi-\tilde{\varphi}\|_{\mathbf{F}_{M}^{s, \sigma}(I)} \\
& +\left(C_{0} \beta^{\theta} B^{1-\theta}+2^{3} C_{0}^{3} \alpha A+2^{2} C_{0} A \alpha \eta^{-1}\right) A^{-1}\|\chi-\tilde{\chi}\|_{\mathbf{F}_{M}^{s, \sigma}(I)} .
\end{aligned}
$$

By fixing $\eta=\left(2^{4} C_{0}^{2}\right)^{-1}$, and choosing $\alpha \leqslant\left(2^{12} C_{0}^{3} A\right)^{-1}$ and $\beta \leqslant\left(2^{4} C_{0} B^{1-\theta}\right)^{-\frac{1}{\theta}}$, we have verified that $\mathcal{T}: S \rightarrow S$ is a contraction, hence it has a fixed point $\left(\varphi, \chi, \phi_{+}\right) \in S$ which is unique in $S$. For later purposes we note that we have chosen the thresholds for $\alpha$ and $\beta$ small enough such that the same conclusion holds if $\alpha, A$, and $B$ are doubled. Similar estimates show that the fixed point depends continuously on the initial data. Due to (4.1), the claimed estimates on the Sobolev norms for $\left(\varphi(t), \chi(t), \phi_{+}(t)\right)$ for $t \in I$ follow from (4.5), (4.6) and (4.7).

Finally, we prove uniqueness. Assume that $\left(\varphi^{\prime}, \chi^{\prime}, \phi_{+}^{\prime}\right) \in \mathbf{F}_{M}^{s, \sigma}(I) \times \mathbf{F}_{M}^{s, \sigma}(I) \times$ $\mathbf{V}_{+, m}^{s+\frac{1}{2}, \sigma}(I)$ is another solution with the same data at $t_{0}$ such that

$$
t^{\prime}:=\sup \left\{t \in I \mid\left(\varphi^{\prime}, \chi^{\prime}, \phi_{+}^{\prime}\right)(t)=\left(\varphi, \chi, \phi_{+}\right)(t)\right\}<t_{2} .
$$

Then,

$$
\left\|\varphi^{\prime}\left(t^{\prime}\right)\right\|_{H_{\sigma}^{s}} \leqslant 2 \alpha, \quad\left\|\chi^{\prime}\left(t^{\prime}\right)\right\|_{H_{\sigma}^{s}} \leqslant 2 A, \quad\left\|\phi_{+}^{\prime}\left(t^{\prime}\right)\right\|_{H_{\sigma}^{\frac{1}{2}+s}} \leqslant 2 B .
$$

Let $\left\|\phi_{+}^{\prime}\right\|_{\mathbf{v}_{+, m}^{s+\frac{1}{2}, \sigma}(I)} \leq R$. By Lemma 4.2 we have

$$
\left\|\phi_{+}^{\prime}\right\|_{\mathbf{D}_{\sigma}^{s}\left(I^{\prime}\right)} \leqslant C_{0}\left\|\phi_{+}^{\prime}\right\|_{\mathbf{V}_{+, m}^{s+\frac{1}{2}, \sigma}\left(I^{\prime}\right)} \leqslant C_{0}^{2} R
$$

for any $I^{\prime} \subseteq I$. For $\varepsilon \in(0, \beta)$ (which will be specified below), let $\delta>0$ be small enough such that $I^{\prime}:=\left[t^{\prime}, t^{\prime}+\delta\right) \subset I$ and $\left\|\phi_{+}^{\prime}\right\|_{\mathbf{D}_{\sigma}^{s}\left(I^{\prime}\right)} \leqslant \varepsilon$. Let $\varphi_{L}^{\prime}(t):=$ $\mathcal{U}_{M}\left(t-t^{\prime}\right) \varphi\left(t^{\prime}\right), \chi_{L}^{\prime}(t):=\mathcal{U}_{M}\left(t-t^{\prime}\right) \chi\left(t^{\prime}\right)$, and $\phi_{+, L}^{\prime}(t):=\mathcal{U}_{m}^{+}\left(t-t^{\prime}\right) \phi_{+}\left(t^{\prime}\right)$. Then,

$$
\left\|\varphi^{\prime}-\varphi_{L}^{\prime}\right\|_{\mathbf{F}_{M}^{s, \sigma}\left(I^{\prime}\right)} \leqslant C_{0} \varepsilon^{\theta} R^{1-\theta}\left(\left\|\varphi^{\prime}-\varphi_{L}^{\prime}\right\|_{\mathbf{F}_{M}^{s, \sigma}\left(I^{\prime}\right)}+\left\|\varphi_{L}^{\prime}\right\|_{\mathbf{F}_{M}^{s, \sigma}\left(I^{\prime}\right)}\right),
$$


so that if we fix some $\varepsilon \leqslant\left(2 C_{0} R^{1-\theta}\right)^{-\frac{1}{\theta}}$, we obtain

$$
\left\|\varphi^{\prime}-\varphi_{L}^{\prime}\right\|_{\mathbf{F}_{M}^{s, \sigma}\left(I^{\prime}\right)} \leqslant\left\|\varphi\left(t^{\prime}\right)\right\|_{H_{\sigma}^{s}} .
$$

A similar estimate shows

$$
\left\|\chi^{\prime}-\chi_{L}^{\prime}\right\|_{\mathbf{F}_{M}^{s, \sigma}\left(I^{\prime}\right)} \leqslant\left\|\chi\left(t^{\prime}\right)\right\|_{H_{\sigma}^{s}} .
$$

Then,

$$
\left\|\phi_{+}^{\prime}-\phi_{+, L}^{\prime}\right\|_{\mathbf{v}_{+, m}^{s+\frac{1}{2}, \sigma}\left(I^{\prime}\right)} \leqslant 2^{3} C_{0}\left\|\varphi\left(t^{\prime}\right)\right\|_{H_{\sigma}^{s}}\left\|\chi\left(t^{\prime}\right)\right\|_{H_{\sigma}^{s}} .
$$

These estimates show that $\left(\varphi^{\prime}, \chi^{\prime}, \phi_{+}^{\prime}\right)$ is contained in the set $S$ defined as above, but with the modified initial condition at $t^{\prime}$ instead of $t_{0}$ and the interval $I^{\prime}$ instead of $I$. Also, the estimates with $I$ replaced by $I^{\prime}$ in the first part of the proof imply that $\left.\left(\varphi, \chi, \phi_{+}\right)\right|_{I^{\prime}}$ is contained in this version of the set $S$. The uniqueness within $S$ proven above implies that $\left(\varphi^{\prime}, \chi^{\prime}, \phi_{+}^{\prime}\right)=\left(\varphi, \chi, \phi_{+}\right)$in $I^{\prime}$, which contradicts the definition of $t^{\prime}$.

We can now prove Theorem 4.1 as follows. By our hypothesis, the initial data at time 0 satisfy

$$
\left\|\varphi_{0}\right\|_{H_{\sigma}^{s}} \leqslant \epsilon,\left\|\chi_{0}\right\|_{H_{\sigma}^{s}} \leqslant A,\left\|\phi_{0}\right\|_{H_{\sigma}^{\frac{1}{2}+s}} \leqslant A,
$$

and $\epsilon>0$ is chosen small enough, depending on $A$ only (the precise threshold will be specified below). Let $\beta^{*}(B)=c B^{\frac{\theta-1}{\theta}}$ and $\alpha^{*}(A)=c A^{-1}$ be the thresholds as in Theorem 4.3. Then, by the Strichartz estimate from Lemma 4.2 (V) , we have

$$
\left\|\mathcal{U}_{m}^{+}(t) \phi_{0}\right\|_{\mathbf{D}_{\sigma}^{s}\left(\mathbb{R}_{+}\right)} \leqslant C_{0} A
$$

with $C_{0} \geqslant 1$. By monotone convergence, the function $T \mapsto\left\|\mathcal{U}_{m}^{+}(t) \phi_{0}\right\|_{\mathbf{D}_{\sigma}^{s}\left(\left[T_{0}, T\right)\right)}$ is continuous in $T$ and converges to zero as $T \searrow T_{0}$. Therefore, for $\beta:=\beta^{*}(2 A)$, we can choose $0=s_{0}<s_{1}<\ldots<s_{N}$ such that

$$
\left\|\mathcal{U}_{m}^{+}(t) \phi_{0}\right\|_{\mathbf{D}_{\sigma}^{s}\left(\left[s_{n-1}, s_{n}\right)\right)}=\beta / 4 \text { and }\left\|\mathcal{U}_{m}^{+}(t) \phi_{0}\right\|_{\mathbf{D}_{\sigma}^{s}\left(\left[s_{n}, \infty\right)\right)} \leqslant \beta / 4
$$

With $s_{N+1}=\infty$, define the collection of intervals $I_{n}=\left[s_{n-1}, s_{n+1}\right)$ for $n=1, \ldots, N$. Then,

$$
\beta / 4 \leqslant\left\|\mathcal{U}_{m}^{+}(t) \phi_{0}\right\|_{\mathbf{D}_{\sigma}^{s}\left(I_{n}\right)} \leqslant \beta / 2
$$

and, by Minkowski's inequality,

$$
\sum_{n=1}^{N}\left\|\mathcal{U}_{m}^{+}(t) \phi_{0}\right\|_{\mathbf{D}_{\sigma}^{s}\left(I_{n}\right)}^{4} \leqslant 2\left(C_{0} A\right)^{4}
$$

therefore $N \leqslant N_{0}:=2^{6}\left(C_{0} A\right)^{4} \beta^{-4}$.

Now, fix $\epsilon \leqslant c C^{-1} C_{0}^{-1} 2^{-2 N_{0}} A^{-1} \beta$. We claim that for every $1 \leqslant n \leqslant N$, on $I_{n}$ we have a unique solution $\left(\varphi^{(n)}, \chi^{(n)}, \phi_{+}^{(n)}\right) \in \mathbf{F}_{M}^{s, \sigma}\left(I_{n}\right) \times \mathbf{F}_{M}^{s, \sigma}\left(I_{n}\right) \times \mathbf{V}_{+, m}^{s+\frac{1}{2}, \sigma}\left(I_{n}\right)$ with initial condition

$$
\begin{aligned}
\left(\varphi^{(n)}, \chi^{(n)}, \phi_{+}^{(n)}\right)\left(s_{n-1}\right) & =\left(\varphi^{(n-1)}, \chi^{(n-1)}, \phi_{+}^{(n-1)}\right)\left(s_{n-1}\right) \quad(\text { if } 2 \leqslant n \leqslant N) \\
\left(\varphi^{(1)}, \chi^{(1)}, \phi_{+}^{(1)}\right)\left(s_{0}\right) & =\left(\varphi_{0}, \chi_{0}, \phi_{0}\right) \quad(\text { if } n=1)
\end{aligned}
$$


which satisfies the bounds

$$
\begin{array}{r}
\left\|\mathcal{U}_{m}^{+}\left(\cdot-s_{n-1}\right) \phi_{+}^{(n-1)}\left(s_{n-1}\right)\right\|_{\mathbf{D}_{\sigma}^{s}\left(I_{n}\right)} \leqslant \beta, \\
\left\|\varphi^{(n)}\left(s_{n}\right)\right\|_{H_{\sigma}^{s}} \leqslant 2^{n} \epsilon, \quad\left\|\chi^{(n)}\left(s_{n}\right)\right\|_{H_{\sigma}^{s}} \leqslant 2^{n} A, \\
\left\|\phi_{+}^{(n)}\left(s_{n}\right)-\mathcal{U}_{m}^{+}\left(s_{n}\right) \phi_{0}\right\|_{H_{\sigma}^{\frac{1}{2}+s}} \leqslant C 2^{2 n} \epsilon A,
\end{array}
$$

where $C$ is the constant from Theorem 4.3. Indeed, for $n=1$ the estimate in the first line follows by definition of $I_{1}$, and the estimates in the second and third line follow from an application of Theorem 4.3 (with $t_{0}=0$ ), where we use that $\epsilon \leqslant \alpha^{*}(A)$ and $\beta \leqslant \beta^{*}(A)$. As an induction hypothesis, let us suppose that holds (4.8) for some $1 \leqslant n \leqslant N-1$.

By Lemma 4.2 the induction hypothesis, and the choice of $\epsilon$ we have

$$
\begin{aligned}
\left\|\mathcal{U}_{m}^{+}\left(\cdot-s_{n}\right) \phi_{+}^{(n)}\left(s_{n}\right)\right\|_{\mathbf{D}_{\sigma}^{s}\left(I_{n+1}\right)} \leqslant & \left\|\mathcal{U}_{m}^{+} \phi_{0}\right\|_{\mathbf{D}_{\sigma}^{s}\left(I_{n+1}\right)} \\
& +\left\|\mathcal{U}_{m}^{+}\left(\phi_{0}-\mathcal{U}_{m}^{+}\left(-s_{n}\right) \phi_{+}^{(n)}\left(s_{n}\right)\right)\right\|_{\mathbf{D}_{\sigma}^{s}\left(I_{n+1}\right)} \\
\leqslant & \beta / 2+C_{0}\left\|\phi_{0}-\mathcal{U}_{m}^{+}\left(-s_{n}\right) \phi_{+}^{(n)}\left(s_{n}\right)\right\|_{H_{\sigma}^{\frac{1}{2}+s}} \\
\leqslant & \beta / 2+C C_{0} 2^{2 n} \epsilon A \leqslant \beta .
\end{aligned}
$$

From the estimate in the third line of the induction hypothesis and the smallness condition on $\epsilon$ we obtain

$$
\left\|\phi_{+}^{(n)}\left(s_{n}\right)\right\|_{H_{\sigma}^{\frac{1}{2}+s}} \leqslant\left\|\mathcal{U}_{m}^{+}\left(s_{n}\right) \phi_{0}\right\|_{H_{\sigma}^{\frac{1}{2}+s}}+C 2^{2 n} \epsilon A \leqslant A+C 2^{2 n} \epsilon A \leqslant 2 A .
$$

Notice that due to our choices we have $\beta \leqslant \beta^{*}(2 A)$ and $2^{n} \epsilon \leqslant \alpha^{*}\left(2^{n} A\right)$. Then, as $s_{n+1} \in I_{n+1}$, we obtain from Theorem 4.3 (with $t_{0}=s_{n}$ ) that

$$
\begin{aligned}
& \left\|\varphi^{(n+1)}\left(s_{n+1}\right)\right\|_{H_{\sigma}^{s}} \leqslant 2\left\|\varphi^{(n)}\left(s_{n}\right)\right\|_{H_{\sigma}^{s}} \leqslant 2^{n+1} \epsilon, \\
& \left\|\chi^{(n+1)}\left(s_{n+1}\right)\right\|_{H_{\sigma}^{s}} \leqslant 2\left\|\chi^{(n)}\left(s_{n}\right)\right\|_{H_{\sigma}^{s}} \leqslant 2^{n+1} A,
\end{aligned}
$$

and, using the induction hypothesis again,

$$
\begin{aligned}
& \left\|\phi_{+}^{(n+1)}\left(s_{n+1}\right)-\mathcal{U}_{m}^{+}\left(s_{n+1}\right) \phi_{0}\right\|_{H_{\sigma}^{\frac{1}{2}+s}} \\
\leqslant & \left\|\phi_{+}^{(n+1)}\left(s_{n+1}\right)-\mathcal{U}_{m}^{+}\left(s_{n+1}-s_{n}\right) \phi_{+}^{(n)}\left(s_{n}\right)\right\|_{H_{\sigma}^{\frac{1}{2}+s}} \\
& +\left\|\mathcal{U}_{m}^{+}\left(s_{n+1}-s_{n}\right) \phi_{+}^{(n)}\left(s_{n}\right)-\mathcal{U}_{m}^{+}\left(s_{n+1}\right) \phi_{0}\right\|_{H_{\sigma}^{\frac{1}{2}+s}} \\
\leqslant & C 2^{2 n} \epsilon A+C 2^{2 n} \epsilon A \leqslant C 2^{2(n+1)} \epsilon A .
\end{aligned}
$$

The proof of the claim is complete.

By uniqueness, we have constructed a global solution

$$
\left(\varphi, \chi, \phi_{+}\right) \in C_{b}\left(\mathbb{R}_{+}, H_{\sigma}^{s}\right) \times C_{b}\left(\mathbb{R}_{+}, H_{\sigma}^{s}\right) \times C_{b}\left(\mathbb{R}_{+}, H_{\sigma}^{\frac{1}{2}+s}\right),
$$

and due to $\left.\left(\varphi, \chi, \phi_{+}\right)\right|_{\left[s_{N}, \infty\right)} \in \mathbf{F}_{M}^{s, \sigma}\left(\left[s_{N}, \infty\right)\right) \times \mathbf{F}_{M}^{s, \sigma}\left(\left[s_{N}, \infty\right)\right) \times \mathbf{V}_{+, m}^{s+\frac{1}{2}, \sigma}\left(\left[s_{N}, \infty\right)\right)$ it scatters as $t \rightarrow \infty$, see Lemma 4.2 Part (iv). The claim for $t \rightarrow-\infty$ follows by time reversibility. Continuous dependence also follows from the local result, we omit the details. This completes the proof of Theorem 4.1 . 


\section{REFERENCES}

1. Alain Bachelot, Global existence of large amplitude solutions for Dirac-Klein-Gordon systems in Minkowski space, Nonlinear hyperbolic problems (Bordeaux, 1988), Lecture Notes in Math., vol. 1402, Springer, Berlin, 1989, pp. 99-113. MR 1033278

2. Global existence of large amplitude solutions for nonlinear massless Dirac equation, Portugal. Math. 46 (1989), no. suppl., 455-473, Workshop on Hyperbolic Systems and Mathematical Physics (Lisbon, 1988). MR 1080766

3. Ioan Bejenaru and Sebastian Herr, The cubic Dirac equation: small initial data in $H^{1}\left(\mathbb{R}^{3}\right)$, Comm. Math. Phys. 335 (2015), no. 1, 43-82. MR 3314499

4. The cubic Dirac equation: small initial data in $H^{\frac{1}{2}}\left(\mathbb{R}^{2}\right)$, Comm. Math. Phys. 343 (2016), no. 2, 515-562. MR 3477346

5. - On global well-posedness and scattering for the massive Dirac-Klein-Gordon system, J. Eur. Math. Soc. (JEMS) 19 (2017), no. 8, 2445-2467. MR 3668064

6. James D. Bjorken and Sidney D. Drell, Relativistic quantum mechanics, McGraw-Hill Book Co., New York-Toronto-London, 1964. MR 0187641

7. Nikolaos Bournaveas and Timothy Candy, Global well-posedness for the massless cubic Dirac equation, Int. Math. Res. Not. IMRN (2016), no. 22, 6735-6828. MR 3632067

8. Timothy Candy and Sebastian Herr, Conditional large data scattering results for the DiracKlein-Gordon system, arXiv, 2017.

9. — Transference of Bilinear Restriction Estimates to Quadratic Variation Norms and the Dirac-Klein-Gordon System, arXiv:1605.04882.

10. John M. Chadam and Robert T. Glassey, On certain global solutions of the Cauchy problem for the (classical) coupled Klein-Gordon-Dirac equations in one and three space dimensions, Arch. Rational Mech. Anal. 54 (1974), 223-237. MR 0369952 (51 \#6181)

11. P. D'Ancona and M. Okamoto, On the cubic Dirac equation with potential and the LochakMajorana condition, arxiv:1706.06479.

12. Steven R. Elliott and Marcel Franz, Colloquium: Majorana fermions in nuclear, particle, and solid-state physics, Rev. Mod. Phys. 87 (2015), 137-163.

13. Georges Lochak, Wave equation for a magnetic monopole, International Journal of Theoretical Physics 24 (1985), no. 10, 1019-1050.

14. Ettore Majorana, Teoria simmetrica dell'elettrone e del positrone, Il Nuovo Cimento 14 (1937), no. 4, 171-184.

15. Tohru Ozawa and Kazuyuki Yamauchi, Structure of Dirac matrices and invariants for nonlinear Dirac equations, Differential Integral Equations 17 (2004), no. 9-10, 971-982. MR 2082456

16. Bernd Thaller, The Dirac equation, Texts and Monographs in Physics, Springer-Verlag, Berlin, 1992. MR 1219537

(T. Candy) Universität Bielefeld, Fakultät für Mathematik, Postfach 100131, 33501 Bielefeld, Germany

E-mail address: tcandy@math.uni-bielefeld.de

(S. Herr) Universität Bielefeld, Fakultät für Mathematik, Postfach 100131, 33501 Bielefeld, Germany

E-mail address: herr@math.uni-bielefeld.de 